\title{
Arcus: exploring the formation and evolution of clusters, galaxies, and stars
}

R. K. Smith, M. Abraham, R. Allured, M. Bautz, J. Bookbinder, et al.

R. K. Smith, M. Abraham, R. Allured, M. Bautz, J. Bookbinder, J. Bregman, L. Brenneman, N. S. Brickhouse, D. Burrows, V. Burwitz, P. N. Cheimets, E. Costantini, S. Dawson, C. DeRoo, A. Falcone, A. R. Foster, L. Gallo, C. E. Grant, H. M. Günther, R. K Heilmann, E. Hertz, B. Hine, D. Huenemoerder, J. S. Kaastra, I. Kreykenbohm, K. K. Madsen, R. McEntaffer, E. Miller, J. Miller, E. Morse, R. Mushotzky, K. Nandra, M. Nowak, F. Paerels, R. Petre, K. Poppenhaeger, A. Ptak, P. Reid, J. Sanders, M. Schattenburg, N. Schulz, A. Smale, P. Temi, L. Valencic, S. Walker, R. Willingale, J. Wilms, S. J. Wolk, "Arcus: exploring the formation and evolution of clusters, galaxies, and stars," Proc. SPIE 10397, UV, X-Ray, and Gamma-Ray Space Instrumentation for Astronomy XX, 103970Q (29 August 2017); doi: $10.1117 / 12.2272818$

Event: SPIE Optical Engineering + Applications, 2017, San Diego, California, United States 


\section{Arcus: The X-ray Grating Spectrometer Explorer}

R. K. Smith ${ }^{* a}$, M. Abraham ${ }^{\text {b }, ~ R . ~ A l l u r e d ~}{ }^{\mathrm{a}}$, M. Bautz ${ }^{\mathrm{c}}$ J. Bookbinder ${ }^{\mathrm{d}}$, J. Bregman ${ }^{\mathrm{e}}$, L. Brenneman $^{\mathrm{a}}$, N.

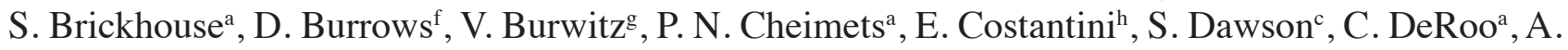
Falcone $^{\mathrm{f}}$, A. R. Foster ${ }^{\mathrm{a}}$, L. Gallo ${ }^{\mathrm{i}}$, C. E. Grant ${ }^{\mathrm{c}}$, H. M. Günther ${ }^{\mathrm{c}}$, R. K. Heilmann ${ }^{\mathrm{c}}$, E. Hertz $^{\mathrm{a}}$, B. Hine ${ }^{\mathrm{d}}$, D. Huenemoerder ${ }^{\mathrm{c}}$, J. S. Kaastra ${ }^{\mathrm{h}}$, I. Kreykenbohm ${ }^{\mathrm{j}}$, K. K. Madsen ${ }^{\mathrm{k}}$, R. McEntaffer ${ }^{\mathrm{f}}$, E. Miller ${ }^{\mathrm{c}}$, J. Miller $^{\mathrm{e}}$, E. Morse ${ }^{\mathrm{l}}$, R. Mushotzky ${ }^{\mathrm{m}}, \mathrm{K}$. Nandra ${ }^{\mathrm{g}}, \mathrm{M}$. Nowak ${ }^{\mathrm{c}}$, F. Paerels ${ }^{\mathrm{n}}$, R. Petre ${ }^{\mathrm{o}}$, K. Poppenhaeger $^{\mathrm{p}}$,

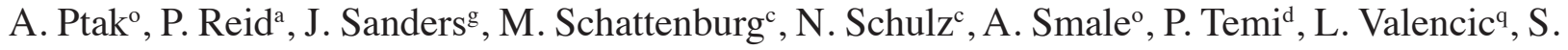
Walker $^{\mathrm{d}}$, R. Willingale ${ }^{\mathrm{r}}$, J. Wilms ${ }^{\mathrm{j}}, \mathrm{S}$. J. Wolk ${ }^{\mathrm{a}}$

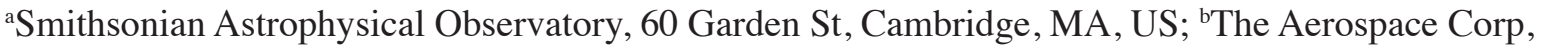

Pasadena, CA, US, ${ }^{c}$ Massachusetts Institute of Technology, Cambridge, MA, US; ${ }^{\text {NASA Ames Research }}$

Center, Moffet Field, CA US; ${ }^{~}$ University of Michigan, Ann Arbor, MI, US; ${ }^{\text {T}}$ The Pennsylvania State

University, University Park, PA, US; ${ }^{9}$ Max-Planck-Institut für extraterrestrische Physik, Garching, DE; hS-

RON Netherlands Institute for Space Research, Utrecht, NL; iSaint Mary's University, Halifax, Canada; jFriedrich-Alexander-Universitaet, Erlangen-Nürnberg, DE; 'California Institute of Technology, Pasadena, CA,

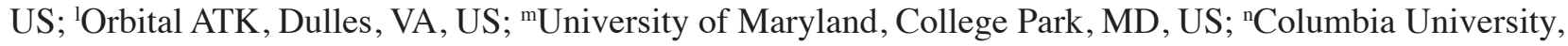
New York, NY, US; ${ }^{\circ}$ NASA Goddard Space Flight Center, Greenbelt, MD, US, PQueen's University, Belfast, UK; ' ${ }^{\mathrm{J} J o h n s}$ Hopkins University, Baltimore, MD, US; ' ${ }^{\mathrm{C}}$ nniversity of Leicester, Leicester, UK

\section{ABSTRACT}

Arcus, a Medium Explorer (MIDEX) mission, was selected by NASA for a Phase A study in August 2017.

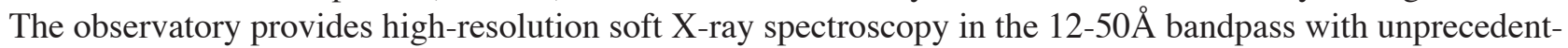
ed sensitivity: effective areas of $>450 \mathrm{~cm}^{2}$ and spectral resolution $>2500$. The Arcus key science goals are (1) to measure the effects of structure formation imprinted upon the hot baryons that are predicted to lie in extended halos around galaxies, groups, and clusters, (2) to trace the propagation of outflowing mass, energy, and momentum from the vicinity of the black hole to extragalactic scales as a measure of their feedback and (3) to explore how stars, circumstellar disks and exoplanet atmospheres form and evolve. Arcus relies upon the same $12 \mathrm{~m}$ focal length grazing-incidence silicon pore X-ray optics (SPO) that ESA has developed for the Athena mission; the focal length is achieved on orbit via an extendable optical bench. The focused X-rays from these optics are diffracted by high-efficiency Critical-Angle Transmission (CAT) gratings, and the results are imaged with flight-proven CCD detectors and electronics. The power and telemetry requirements on the spacecraft are modest. Mission operations are straightforward, as most observations will be long ( $100 \mathrm{ksec})$, uninterrupted, and pre-planned, although there will be capabilities to observe sources such as tidal disruption events or supernovae with a $\sim 3$ day turnaround. Following the $2^{\text {nd }}$ year of operation, Arcus will transition to a proposal-driven guest observatory facility.

Keywords: Gratings, X-rays: spectroscopy, Instrumentation

\section{INTRODUCTION}

In 2014, NASA's Astrophysics Science Mission Directorate described their plans ${ }^{\S}$ to "explore the origin and evolution of the galaxies, stars and planets that make up our Universe" - i.e., to investigate structure formation across all mass scales. The formation of galaxies, groups, and clusters leaves a major fraction of the input gas in extended halos around these structures. This gas, which represents the majority of baryonic matter in the Universe, experiences shock heating, radiative cooling and powerful winds from black holes and star formation. These processes leave the gas in the intergalactic medium hot $\left(\mathrm{T} \sim 10^{5}-10^{8} \mathrm{~K}\right)$, tenuous and metal-enriched. These signatures of structure formation are found in the resulting spatial distribution of this vol-

"rsmith@cfa.harvard.edu; phone 1617 495-7143; http://hea-www.harvard.edu/ rsmith; http://www.arcusxray.org shttps://science.nasa.gov/about-us/science-strategy

UV, X-Ray, and Gamma-Ray Space Instrumentation for Astronomy XX, edited by Oswald H. Siegmund, Proc. of SPIE Vol. 10397, 103970Q - @ 2017 SPIE · CCC code: 0277-786X/17/\$18 · doi: 10.1117/12.2272818 
ume-filling gas, its composition, and its motions. Observational efforts with existing telescopes have proven inadequate to detect these signatures. The bulk of this hot gas and most of the metals in the Universe have not been detected, a shortcoming Arcus will resolve.

Energy emitted by supermassive black holes and rapid star formation in the form of winds and jets interact with this hot gas in a process termed "feedback." Characterizing its underlying mechanisms requires tracing the propagation of outflowing mass, energy, and momentum from the vicinity of the black hole out to megaparsec scales. Similar physics also occurs on smaller scales, in the form of stellar accretion, stellar coronae, and galactic structure. Our ability to understand these processes and many others

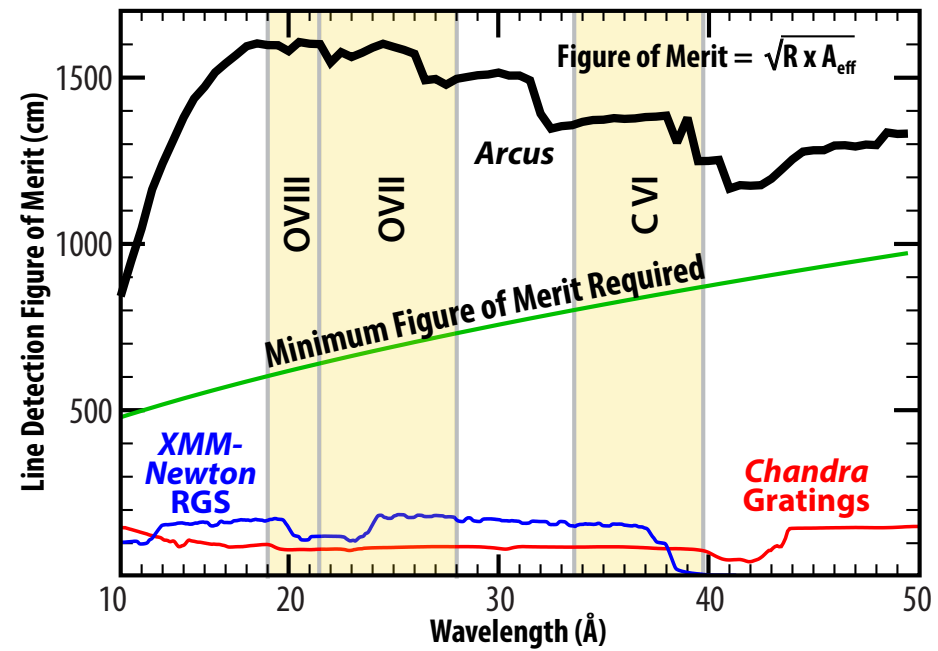

Figure 1: Only Arcus has the combination of sensitivity and resolution needed to detect weak absorption features that reveal the "missing baryons" at redshifts $<0.3$ (yellow bands). The green line shows the requirement for detection of a $3 \mathrm{~mA}$ equivalent width feature at $5 \sigma$ in $500 \mathrm{ks}$ using a distant supermassive black hole as a background source.

from every abundant metal. High-resolution $\mathrm{X}$-ray spectroscopy has already led to major astronomical discoveries $[1,2,3,4,5]$ but potential progress has been limited both by low effective areas, typically $<10 \%$ of X-ray imaging instruments, and limited resolutions. Neither the Chandra nor XMM-Newton gratings provide the sensitivity required to effectively survey the hot low density gas at and beyond the outskirts of galaxies and clusters, despite the fact that most of the baryons in the Universe are found in these regions. As shown in Figure 1, the detection of this matter, as well as answers to the other questions listed above, requires the major leap in spectral resolution and sensitivity that Arcus has been specifically designed to provide.

Arcus leverages both NASA's investment in the development of high-resolution, efficient lightweight gratings and ESA's development of silicon pore optics. Combining these technologies provides order of magnitude or larger improvements in collecting area and spectral resolution compared to existing spectrometers, with a commensurate increase in the relevant Figure of Merit in the key O VII/O VIII bandpass (see Figure 1). Flight-proven Suzaku-based CCDs and Swift-based instrument electronics are strong heritage components, while the spacecraft and mission operations reuse highly successful approaches. Arcus' design philosophy built significant internal subsystem redundancies in optics and detectors, providing robust cost and schedule reserve. Equally important, the experienced team has been involved in the planning, development, delivery, and operation of virtually every successful U.S. X-ray mission of the last three decades.

The Arcus grating instrument complements the calorimeters on JAXA's XARM and ESA's Athena missions, the next planned X-ray satellites with high spectral resolution capabilities. The grating spectral resolution will be more than an order of magnitude higher than a calorimeter in the soft $(<1 \mathrm{keV}) \mathrm{X}$-ray band, enabling science investigations not otherwise possible. By tracing diffuse hot gas, Arcus will also complement the next generation of powerful ground and space observatories (e.g., LSST, ALMA, JWST and Euclid), which will use gravitational lensing and galaxy surveys to trace galaxy and dark matter distributions and cold gas in galaxies. Arcus will address fundamental questions posed by the 2010 Decadal Survey, such as "How/why does ordinary matter assemble into the large-scale structures that we see today?" and "How does black hole feedback influence the Universe?" 


\section{SCIENCE}

The Arcus science case [8] covers a range of questions raised in the 2010 Decadal survey. Over its two year baseline mission, Arcus will:

1) Measure the spatial and temperature distribution of hot gas at and beyond the virial radii of galaxies and clusters, and the distribution and metal abundance of all phases of gas and dust in our Galaxy's disk. Hot halos of gas surrounding galaxies and clusters can be studied in detail by measuring their X-ray absorption properties against the continua of bright background sources. Measuring the properties of this absorbing gas will provide the first complete picture of the formation and cycling of metals in and out of galaxies and clusters.

2) Measure the mass, energy and composition of outflowing winds from the inner regions of supermassive and stellar mass black holes. Actively-feeding black holes (active galactic nuclei, or AGN) produce X-ray continuum emission that is absorbed by the outflowing hot gas. Characterizing this absorption will reveal the ionization state, density and total gas mass being carried away by such outflows. Measuring these properties will shed light on how black holes power winds and how the winds interact with cooler gas in the surrounding galaxy and beyond, seeding it with energy and ultimately regulating star formation.

3) Measure the thermodynamic properties of hot gas in stellar magnetic structures and shocks. Coronal $\mathrm{X}$-ray emission scales with stellar rotation, revealing the actions of internal magnetic dynamos at work. Measuring emission line fluxes from a statistical sample of stars will determine their coronal temperatures, allowing the exploration of how the heating of the stellar corona evolves as a star's rotation slows with age. Arcus will also probe the final stages of star formation through observations of accretion from a protoplanetary disk onto the star. The accreting material creates a shock front near the stellar surface, producing characteristic soft X-ray emission that will illuminate how the accretion streams interact with stellar magnetic fields.

Figure 2 shows one aspect of the Arcus science case, using X-ray spectra to measure Galactic dust properties by resolving the composition-specific X-ray absorption fine structures (XAFS) and enabling direct measurements of the chemical composition of the dust [7,8]. XAFS are seen from all dust-forming elements; for example, different forms of carbon grains will have distinct detailed signatures in the soft X-ray bandpass [9]. Spectra from Arcus will allow us to distinguish between models by revealing grain composition and the total abundances of major dust-forming elements via edge shape and depth, respectively. Grain composition is of particular importance to cosmic microwave background polarization measurements [10], as the foreground dust emission is sensitive to the presence of magnetic inclusions [11,12]. In the microwave band, the polarized dust emission varies by a factor of four depending on whether the magnetic inclusions $\operatorname{are~} \mathrm{Fe}_{2} \mathrm{O}_{3}, \mathrm{Fe}_{3} \mathrm{O}_{4}$, or metallic Fe [12]. Only Arcus, of any current or planned mission, has the sensitivity and soft X-ray resolution to clearly distinguish between these dust types using XAFS at the Fe L edge, and thus to determine the expected foreground linear polarization.

\section{INSTRUMENTATION}

\subsection{Overview}

The Arcus (from the Latin for arc, as in a rainbow) science case requires a bandpass from 12-50 , an

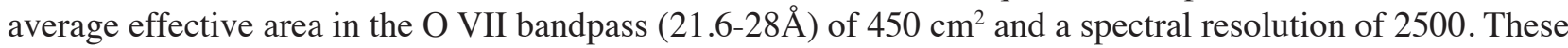
requirements are achieved (see Figure 3 ) using a design combining grazing-incidence X-ray optics with 


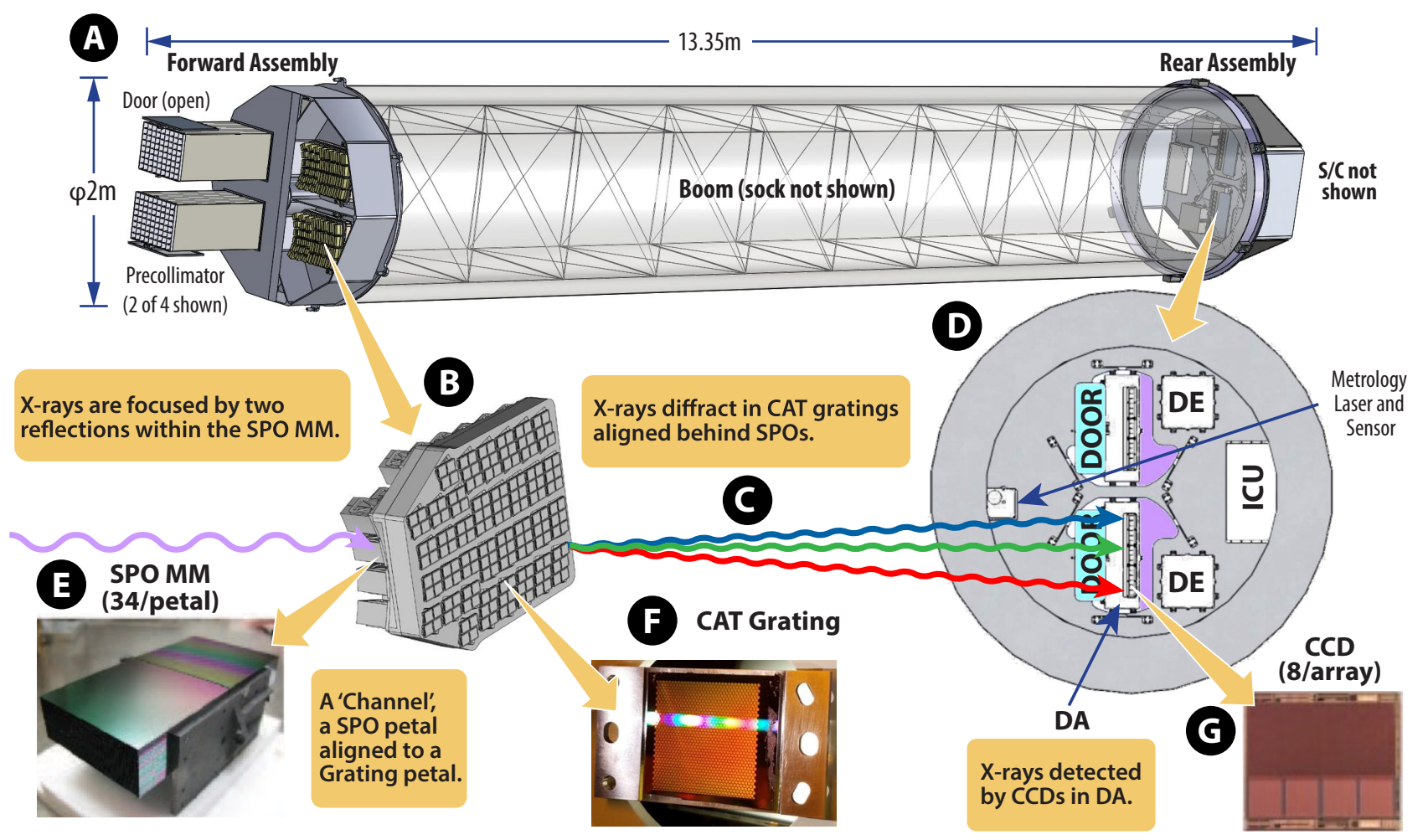

Figure 3: The Arcus overall design and instrumentation; see text for acronym definition.

transmission gratings that disperse incoming photons onto an array of CCD detectors. The optics and detectors are held in position by an Extendable Optical Bench (EOB) whose alignment is monitored by an metrology system $[27,28]$. The design is highly modular, with the optics and gratings arranged into four distinct 300x500mm "petals;" each has a thermal precollimator to reduce thermal losses. A single optic petal and grating petal are aligned to form a "channel" which disperses X-rays onto the focal plane. Two detector arrays are positioned in the focal plane and the system aligned so that each array detects light from two different channels.

\subsection{Silicon Pore Optics}

The Silicon Pore Optics (SPOs) [13, 31] that are being developed by ESA and cosine Measurement Systems for the ESA Athena mission provide an automated and modular production system that combines high efficiency with modest expense. SPOs have already undergone substantial environmental testing for vibration, shock, and thermal response in preparation for Athena [14]. The Athena and Arcus missions have similar alignment requirements as well. To minimize cost and schedule risk associated with the SPO manufacturing process, Arcus uses the exact same focal length as Athena $(12 \mathrm{~m})$ and similar radii of curvature $(348-784 \mathrm{~mm}$, e.g. rows 2-9 of reference [15]). The size of each SPO Mirror Module (MM) varies slightly with radius, ranging from $76-168 \mathrm{~mm}$ in length with a geometric area facing the sky of $55.8 \mathrm{~mm}$ by $50-90 \mathrm{~mm}$. The current design uses stacks of 35 silicon plates that are $0.775 \mathrm{~mm}$ thick; the grooves that form the pores are $0.605 \mathrm{~mm}$ deep and $2.4 \mathrm{~mm}$ wide, with $0.14 \mathrm{~mm}$ 'ribs,' leading to a total open area of $73.8 \%$.

Thanks to support from the Max Planck Institute for Extraterrestrial Physics, our team obtained the first $12 \mathrm{~m}$ focal length SPO ever produced, with a radius of curvature of $737 \mathrm{~mm}$, although since then SPOs with radii of curvature as small as $250 \mathrm{~mm}$ have been produced [33]. The X-ray performance of the first $12 \mathrm{~m}$ focal length optic (Figure 4) was tested at NASA/Marshall Space Flight Center's Stray Light Facility with support from NASA/MSFC, ESA, and cosine; details are described in [29].

The system's optical design takes advantage of the highly asymmetric nature of the point spread function of modular X-ray optics (see Figure 4) via a method known as "subaperturing" [16,17]. The gratings are aligned 


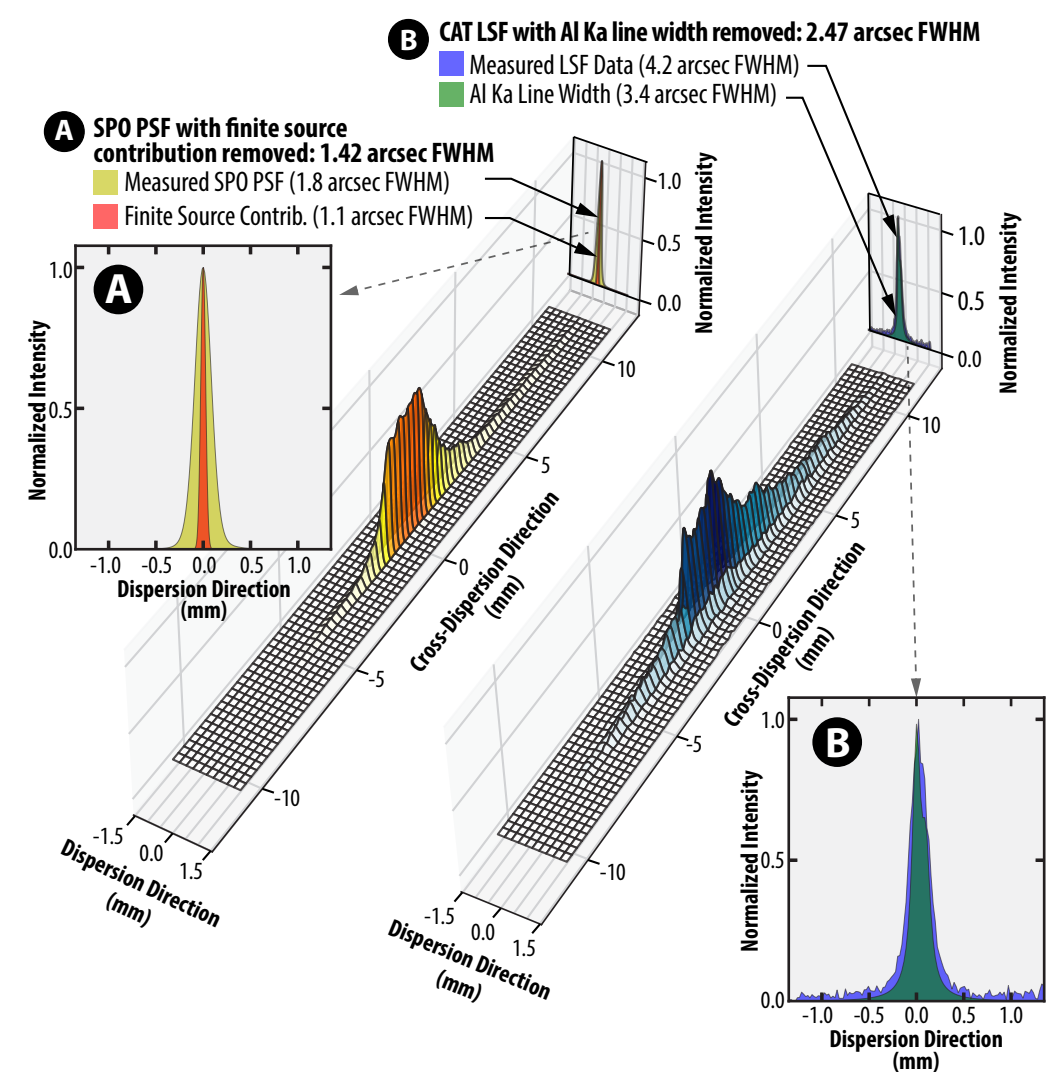

Figure 4: : Results from SPO and CAT grating tests at the NASA Marshall Space Flight Center's Stray Light Facility. so that they disperse the photons along the narrow PSF direction. The width of the other PSF direction then makes the dispersed spectrum broader than it would be if the PSF were narrow in all axes, but this does not substantially interfere with the resolution. Currently, the smallest measured total SPO PSF (for a $20 \mathrm{~m}$ f.l. optic) is 13.9 arc seconds HEW in total [18], but the key value is the narrow axis. As shown in Figure 4(A), the tests at the Stray Light Facility found a total PSF of 1.8 arc seconds FWHM, or 1.42 arc seconds FWHM after removing the 1.1 arc second finite source contribution. The requirement, as shown in Table 1, is 2 arc seconds FWHM, so the first-ever produced $12 \mathrm{~m}$ SPO optic already meets Arcus' needs. Since the initial tests, the $12 \mathrm{~m}$ SPO has been re-tested for confirmation at PANTER with similar results [29].

\subsection{Critical-Angle Transmission} (CAT) Gratings

The Arcus CAT gratings are described in detail in [29] and only summarized

here. CAT gratings were selected for Arcus because of their extremely high resolving power combined with high throughput. They are lightweight, and they are easily aligned to Arcus' requirements [19, 20, 21, 22].

A CAT grating comprises $\sim 130 \mathrm{~nm}$ wide perforations every $200 \mathrm{~nm}$, cut perpendicularly through a $\sim 4$ micron thick Si device layer, leaving $~ 70 \mathrm{~nm}$ wide "walls" between perforations [26]. CAT gratings have been made with sizes of $10 \times 32 \mathrm{~mm}$ and $32 \times 32 \mathrm{~mm}[26,29]$. The exact size of each grating can easily be varied within this range while meeting Arcus' requirements, allowing flexibility in the grating petal design; the current design uses $23 \times 26 \mathrm{~mm}$. The gratings are tilted at $1.9^{\circ}$ from normal incidence, resulting in grazing reflection off the inside walls and enhanced grating efficiency ("blazing") around the direction of the specular reflection. Optimum blazing is set at $21.6-28 \AA$ in 5 th-6th order. The CAT grating ruling density is 5000 lines $/ \mathrm{mm}$, providing a dispersion of $6 \mathrm{~mm} / \AA$ in first order, so the CAT gratings achieve a resolution of $>2500$ in the key 21.6-28 ̊ band with substantial $(\mathrm{R}>4000)$ design margins.

Individual gratings are attached to a Ti window support that is aligned and mounted onto the grating petal. There are four bonding pads for each grating membrane, each at the center point of a flexure. The flexured design isolates the grating from the thermal effects of CTE mismatch with the Ti window, as confirmed with thermal environmental testing. The gratings are very weakly sensitive to alignment errors in pitch, but more sensitive to roll and yaw errors (Table 1). The alignment method itself is described in $[29,30]$.

The initial results from the SLF testing of the SPO with a single 10x32mm CAT grating are described in detail in [29], along with subsequent testing of a pair of aligned CAT gratings done at PANTER with the same SPO.

Figure 4(B) shows the performance of a CAT grating dispersing an Al Ka line. After taking into account the finite source size of the SLF and the natural linewidth of the $\mathrm{Al} \mathrm{K \alpha}$ source, the tests demonstrated a 1.7 arc second LSF for the CAT grating itself, substantially better than the 2.3 arc second requirement shown in Table 1. 


\subsection{Mounting and Aligning the SPO and CAT Petals}

Table 1: Arcus static alignment requirements

The alignment sensitivities were derived using the methods described in [24]. The tightest tolerances are the 12.5 micron relative lateral translations of the SPO modules. The SPO modules will be aligned in the petal structure using a collimat- SPO ed $218 \mathrm{~nm}$ UV beam that simultaneously illuminates multiple modules $[31,32]$. Testing has demonstrated that the UV and $\mathrm{X}$-ray centroids can be measured to within $<0.14$ arcsec, well below the requirements shown in Table 1 [32]. The collimated beam is translated as modules are added in order to maintain alignment across the entire petal. A removable reference flat between the petal and the laser is used to maintain alignment of the collimated beam as it is translated. The procedures for CAT grating alignment are described in $[29,30]$.

\begin{tabular}{|c|c|c|c|c|c|c|c|}
\hline Area & Term & Unit & $\begin{array}{l}\text { Requir- } \\
\text { ement }\end{array}$ & $\begin{array}{l}\text { Capa- } \\
\text { bility }\end{array}$ & $\frac{\text { Req-Cap }}{\text { Cap }}$ & $\begin{array}{l}\text { Impact } \\
\text { on LSF }{ }^{1}\end{array}$ & $\begin{array}{l}\text { Basis } \\
\text { of Est. }\end{array}$ \\
\hline \multirow{4}{*}{ SPO } & Transverse PSF & $\operatorname{arcsec}$ & 2.0 & 1.42 & $40 \%$ & $1.42^{\prime \prime}$ & see Fig. 4 \\
\hline & Align (lateral) & $\mu \mathrm{m}$ & 12.5 & 6.0 & $108 \%$ & $0.10^{\prime \prime}$ & Measured \\
\hline & Align (roll) & $\operatorname{arcsec}$ & 10 & 5 & $100 \%$ & $0.35^{\prime \prime}$ & Measured \\
\hline & Align (pitch/yaw) & $\operatorname{arcsec}$ & 180 & 90 & $100 \%$ & $0.07^{\prime \prime}$ & Measured \\
\hline \multirow{3}{*}{ CAT } & Align (to window) & $\operatorname{arcmin}$ & 5 & 3 & $67 \%$ & $0.24^{\prime \prime}$ & $\operatorname{Ref}[29,30]$ \\
\hline & Align (to petal) & $\operatorname{arcmin}$ & 5 & 3 & $67 \%$ & $0.24^{\prime \prime}$ & $\operatorname{Ref}[29,30]$ \\
\hline & Gratings LSF & $\operatorname{arcsec}$ & 2.3 & 1.7 & $35 \%$ & $1.7^{\prime \prime}$ & see Fig. 4 \\
\hline \multirow{3}{*}{ Channel } & Astigmatism & $\operatorname{arcsec}$ & 2.0 & 1.49 & $34 \%$ & $1.49^{\prime \prime}$ & Modeled \\
\hline & Alignment & $\operatorname{arcmin}$ & 5 & 3 & $67 \%$ & $0.32^{\prime \prime}$ & Chandra \\
\hline & Focus & $\mathrm{mm}$ & 13 & 1 & $1200 \%$ & $0.01^{\prime \prime}$ & Hinode \\
\hline Total & RSS & & $3.76^{\prime \prime}$ & & & $2.74^{\prime \prime}$ & \\
\hline
\end{tabular}

\subsection{Focal Plane and Electronics}

The Arcus detectors consist of two CCD detector arrays (DAs), each with eight 2048x1024 CCD devices and each served by dedicated low-noise detector electronics (DE) connected to the Arcus Instrument Control Unit (ICU). The two DAs operate independently and in parallel, each receiving X-rays from two of the channels. The detector subsystem architecture and its major components have heritage from Chandra and Suzaku and all critical elements in the detector string (DA, DE) have been prototyped and tested. The MIT/LincolnLaboratory-produced CCDs leverage designs with substantial flight heritage. The DA provides $10 \mathrm{gm} \mathrm{cm}^{-2}$ shielding, the CCDs are passively cooled to $\leq-90 \mathrm{C}$, and like Suzaku controlled to $\pm 0.5 \mathrm{C}$, ensuring adequate spectral resolution for order-separation throughout the mission.

The Focal Plane (FP) array (see Figure 3) in each of the DMs comprises eight CCDs to record two dispersed spectra, as well as the undispersed zero order flux, from two different petals. This is achieved by using opposite blaze angles on the two petals, so that each throws the spectra in the correct direction. Each focal plane module is fitted with a filter and door assembly. Primary UV/optical blocking will be provided by an onchip 40nm Al deposition [25]. Additional blocking and contamination protection will be provided by a $45 \mathrm{~nm}$ polyimide filter with a $30 \mathrm{~nm} \mathrm{Al}$ deposition. The door is designed to be opened once upon deployment.

The ICU supplies regulated and unregulated switched power to the instrument, processes instrument commands, records instrument housekeeping, extracts X-ray events from the CCD camera data stream, and formats telemetry. The ICU will be built by Southwest Research Institute (SwRI) and has heritage from $M M S$ and Juno. Event recognition processing will be done via a high-speed Virtex V5 FPGA, which stores bias images, subtracts bias levels, masks bad pixels, searches for local maxima, and extracts $3 \times 3$ event neighborhoods around candidate events. After processing, valid X-ray events will be formatted and sent to the spacecraft via a MILSTD-1553 bus.

Order-sorting of dispersed spectra using the CCD energy resolution measured for each event strongly rejects background, since there is only a narrow range of allowed energies for any order at any dispersion angle. Analysis of the Chandra/HETG background* indicates that nearly all the background is from Galactic cosmic ray protons (i.e., not cosmic X-rays), with a very low rate of about 0.4 counts/Ms per extraction cell, where the extraction cell is one FWHM in the dispersion direction, covers the cross-dispersion width, and

Table 2: Focal Plane Overview

\begin{tabular}{|c|c|}
\hline Characteristic & Value \\
\hline Detectors & Back-illuminated frame transfer X-ray CCDs \\
\hline CCD format & $\begin{array}{l}2048 \times 1024,24 \mu \mathrm{m} \text { pixels }[0.41 \mathrm{arcsec} / \text { pixel], } \\
8 \text { parallel outputs, chip gaps }<500 \mu \mathrm{m}\end{array}$ \\
\hline Focal plane array & $8 C C D s$ per $D A$ \\
\hline CCD spectral resolution & $\Delta \mathrm{E}(\mathrm{FWHM})<70 \mathrm{eV}$ at $\lambda=24 \AA(0.5 \mathrm{keV})$ \\
\hline System read noise & $<4$ e- RMS @ 625 kpixels s $^{-1}$ \\
\hline$C C D$ frame rate & $1 \mathrm{~Hz}$ (ensuring negligible pile-up ) \\
\hline Focal plane temperature & $\leq-90$, stable to $\pm 0.5^{\circ} \mathrm{C}$ (passively cooled) \\
\hline \multirow{2}{*}{$\begin{array}{l}\text { Blocking filters (UV/optical and } \\
\text { molecular contamination) }\end{array}$} & On chip: 40nm Al \\
\hline & On mesh: $45 \mathrm{~nm}$ polyimide plus $30 \mathrm{~nm} \mathrm{Al}$ \\
\hline
\end{tabular}

*http://space.mit.edu/cxc/docs/hetg_bg/ 
is about $100 \mathrm{eV}$ wide in the CCD energy response (an "order-sorting cell"), or for Chandra/ACIS, 15 pixel $^{2}$. Scaling to Arcus with plausible assumptions of similar CCDs, radiation environment, and shielding combined with a $12 \times 50$ pixel spatial extraction cell, $100 \mathrm{eV} \mathrm{CCD}$ width, we obtain 15 counts/Ms per cell, or at $24 \AA ̊$ in 5th order, $1.5 \times 10^{-3}$ cts s$^{-1} \AA^{-1}$, ten times below our required background level.

\subsection{Overview}

\section{MISSION DESIGN}

Arcus' X-ray spectroscopy requires a space mission as X-rays are absorbed by the Earth's atmosphere. To meet the requirements for long observations from a stable platform, Arcus uses a lunar resonant, 4:1 High Earth Orbit (HEO) (see Figure 5) and selects a high-heritage Orbital ATK (OATK) space-craft (S/C) that meets all instrument requirements with robust margins. Arcus is $4 \mathrm{~m}$ high with a $2 \mathrm{~m}$ diameter at launch with a total mass of $1142 \mathrm{~kg}$, comfortably fitting within the capabilities of the standard launch vehicle services provided for MIDEX missions. The mission design enables continuous instrument operations, and the 6.85-day period HEO provides a low radiation environment, benign disturbance torques, and long-term orbit stability, which enables $\mathrm{S} / \mathrm{C}$ accommodation of the instrument with flight-proven technologies and components. Science operations are simple, with long, low-jitter, inertially-pointed observations that are interrupted only by Reaction Wheel (RW) desaturations. No pointing is required for the Deep Space Network (DSN) contacts for the Science Data Downlink (SDDL), which occurs once per orbit near perigee, and includes commanding. The simplicity of operations in the HEO environment leads to a high ( $>85 \%)$ Observation Efficiency ensuring that all science observations can be accomplished within a 2-year baseline science phase.

\subsection{Spacecraft}

The Arcus flight system is designed to meet the mission requirements with robust margins while maximizing heritage and operational flexibility. Arcus uses the LEOStar-2 S/C bus as its core and the basis for its ADCS, C\&DH, Telecom, Power, and Thermal subsystems. This bus is also used by NuSTAR with a similar instrument configuration and adapted for the TESS mission to the HEO environment. The two-year baseline science mission (not including commissioning) includes a goal for an extended mission of up to five years; the flight system consumables are sized for ten years. The spacecraft will provide at least $405 \mathrm{~W}$ continuous power to the instrument, with batteries to survive periodic eclipse periods (during which the instrument will be turned off and operating in survival mode.) The spacecraft will be thermally isolated from the instrument, which is connected to the focal plane support structure.

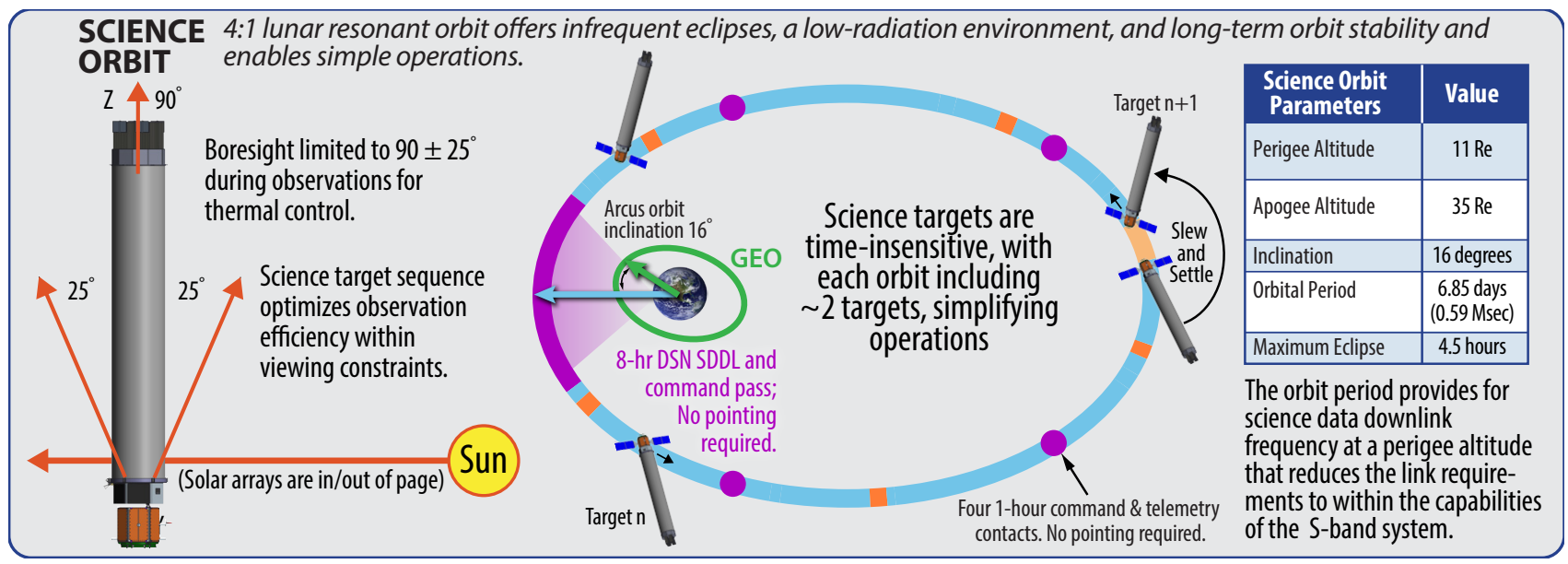

Figure 5: Arcus science orbit and telemetry. The high orbit enables efficient observation scheduling, interrupted only by slewing, settling, and reaction wheel desaturations. Science data downlink (SDDL) is done once per orbit and does not interrupt observing. 


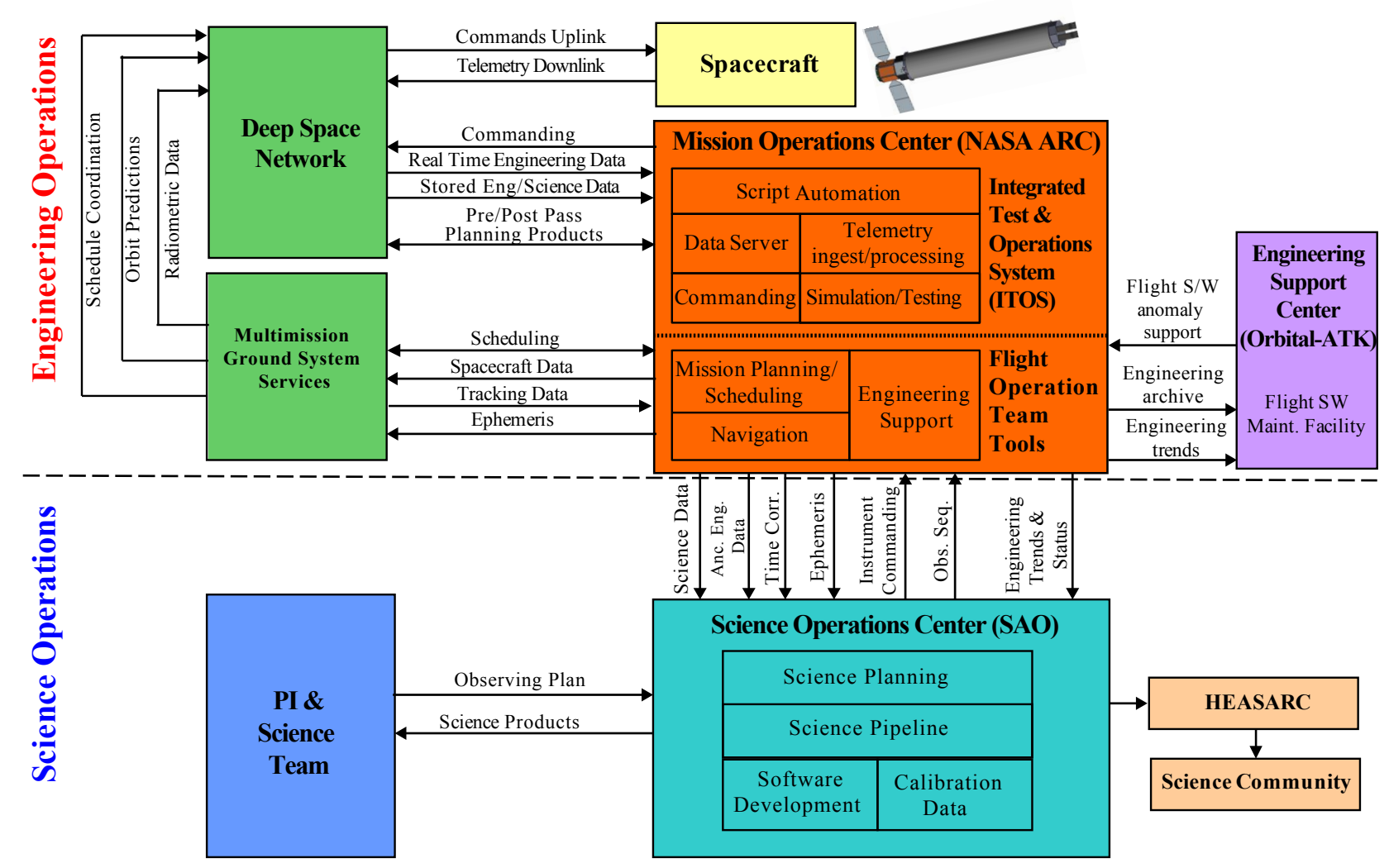

Figure 6: Arcus Operations conceptual flow diagram

\subsection{Overview}

\section{OPERATIONS}

Arcus will operate in a 4:1 lunar resonant $(80,000 \mathrm{~km} \times 225,000 \mathrm{~km})$ orbit, as shown in Figure 5. This stable orbit provides a low radiation environment as it remains above the Van Allen belts at all times, and it also has low disturbance torques from either the Earth or Moon. It provides over $85 \%$ availability for science observations and can be arranged to have rare and short ( $<5$ hour) eclipses.

The orbit provides 6.85 days of science data collection, with 8 hours of science data downlink during perigee passes via one of two low-gain S-band antennae. Arcus will also have four S-band command and telemetry contacts per orbit, which would enable changes to the observing plan on a $\sim 3$ day timescale if a compelling Target of Opportunity is identified.

Mission operations during the baseline mission will benefit from a well-defined target list $(\sim 200$ sources in 2 years) that have no timing or continuity requirements. Similarly, science operations will benefit from strong heritage in software algorithms as well as team members from Chandra and XMM-Newton. An overview of the operational concept is shown in Figure 6.

\subsection{Mission Operations}

NASA Ames Research Center (ARC) will host the Arcus Mission Operations Center (MOC). After commissioning, real-time contacts with the spacecraft will be performed roughly once per week to upload a 2-week long observation schedule. Prior to contacts, command sequences will be verified with ground software, and as needed, validated by simulation on the engineering model. During contacts, the communication configuration and $\mathrm{S} / \mathrm{C}$ and payload state of health are ensured and new tables and command sequences are uplinked as necessary.

On downlinks, upon data receipt, the MOC ground data system will automatically initiate Level 0 processing - reconstructed, time-ordered, unprocessed data with communications artifacts (e.g. sync frames, commu- 
nications headers, duplicate data) removed - and will route pertinent data as appropriate (e.g., instrument data to the Science Operations Center [SOC]).

For staffing purposes, a flight controller will be on call at all times in case of issues with the spacecraft, and will respond according to a previously defined response type. Along with interfacing with the Arcus SOC, the ARC MOC supports local archive of spacecraft telemetry for the duration of Arcus' full and extended mission (5 years; estimated $<1 \mathrm{~Tb}$ ).

\subsection{Science Operations}

The Science Operations Center (SOC) will be located at the Smithsonian Astrophysical Observatory with components at MIT and Friedrich-Alexander University (FAU). The SOC will selects targets along with their observing times and modes, and will process and calibrate the data returned to enable analysis by the full science team. The raw telemetry data received from the MOC will be processed at FAU to create basic X-ray Level 1 event files as well as instrument housekeeping files, using software developed for the eRosita instrument on Spectrum-X Gamma. These files will then be processed to attach grating information to each event (Level 1.5), requiring the identification of dispersed arcs, event orders, and exact wavelengths to tag each event. The algorithms required will be created by the same MIT team that developed Chandra's High Energy Transmission Grating (HETG), using similar approaches as that software. Finally, fully-processed Level 2 event files including identification of background events and bad pixels along with calibrated responses will be generated, again using algorithms and approaches originally developed for Chandra, XMM-Newton, and Suzaku.

The SOC will develop the long and medium-term observation plans. The initial targets were selected as part of the proposal itself, but the team will take into account results from the analysis of early data to ensure the baseline science requirements are addressed. The SOC will also be responsible for maintaining and analyzing a trend database, checking data for short- and long-term trends due to particle environment, contamination, and other effects such as boom bending. Finally, the SOC will be responsible for overall Arcus calibration, including generating all necessary calibration data, offsets, and/or corrections to use in both the MOC command sequence (uplink) and the data analysis tools (downlink). This includes not only the basic pipeline processing that converts raw telemetry data into Level 2 event files, but also all necessary calibration files and tools as well as delivery to the science team and the High Energy Astrophysics Science Archive Research Center (HEASARC) archive.

\section{ACKNOWLEDGEMENTS}

The research leading to these results has in part received funding from the Smithsonian Astrophysical Observatory and the European Union's Horizon 2020 Program under the AHEAD project (grant agreement $\mathrm{n}$. 654215). The Arcus team especially thanks the team at NASA's Marshall Space Flight Center's Stray Light Center for hosting the SPO and CAT testing, including substantial assistance with the data collection and analysis.

\section{REFERENCES}

[1] Paerels, F. \& Kahn, S., "High-Resolution X-Ray Spectroscopy with CHANDRA and XMM-NEWTON", ARA\&A, 41, 291-342 (2003)

[2] Bregman, J. \& Lloyd-Davies, E., "X-Ray Absorption from the Milky Way Halo and the Local Group", ApJ, 669, 990-1002 (2007)

[3] Detmers, R. et al., "Multiwavelength campaign on Mrk 509. III. The 600 ks RGS spectrum: unravelling the inner region of an AGN", A\&A, 534, A38 (2011)

[4] Fang, T. et al., "Confirmation of X-ray Absorption by Warm-Hot Intergalactic Medium in the Sculptor Wall”, ApJ, 714, 1715-1724 (2010)

[5] Brickhouse, N. et al., "X-Ray Determination of the Variable Rate of Mass Accretion onto TW Hydrae", ApJL, 760, 21-26 (2012) 
[6] Brenneman, L. et al., "The evolution of structure and feedback with Arcus," in Society of Photo-Optical Instrumentation Engineers (SPIE) Conference Series, 2016, p. 99054P

[7] Lee, J.C. and Ravel, B., "Determining the Grain Composition of the Interstellar Medium with HighResolution X-Ray Spectroscopy,” The Astrophysical Journal, vol. 622, pp. 970-976, 2005.

[8] Lee, J.C. et al., "Condensed Matter Astrophysics: A Prescription for Determining the Species-specific Composition and Quantity of Interstellar Dust Using X-rays,” The Astrophysical Journal, vol. 702, pp. 970-979, 2009

[9] Draine, B.T., "Graphite Revisited," The Astrophysical Journal, vol. 831, p. 109, 2016.

[10] Barkats, D. et al., "Degree-scale Cosmic Microwave Background Polarization Measurements from Three Years of BICEP1 Data," The Astrophysical Journal, vol. 783, p. 67, 2014.

[11] Draine, B.T. and Fraisse, A.A., "Polarized Far-Infrared and Submillimeter Emission from Interstellar Dust," The Astrophysical Journal, vol. 696, pp. 1-11, 2009.

[12] Draine, B.T. and Hensley, B., "Magnetic Nanoparticles in the Interstellar Medium: Emission Spectrum and Polarization," The Astrophysical Journal, vol. 765, p. 159, 2013.

[13] Bavdaz, M. et al. 2015, "The Athena Optics," in Society of Photo-Optical Instrumentation Engineers (SPIE) Conference Series, 2015, vol.9603, p. 96030J

[14] Wille, E. et al., "Qualification of Silicon Pore Optics," in in Society of Photo-Optical Instrumentation Engineers (SPIE) Conference Series, 2014, p. 91442H

[15] Willingale, R. et al., "Science requirements and optimization of the silicon pore optics design for the Athena mirror," in Society of Photo-Optical Instrumentation Engineers (SPIE) Conference Series, 2014, p. $91442 \mathrm{E}$

[16] Cash, W., "X-ray optics - A technique for high resolution imaging”, Applied Optics, 26, 2915-2920 (1987)

[17] Allured, R et al., "Optical design considerations and raytracing results for the Arcus grating spectrometer concept," in Society of Photo-Optical Instrumentation Engineers (SPIE) Conference Series, 2016, vol 9905, p. 990540

[18] Collon, M. J. et al., "Silicon Pore Optics for the ATHENA telescope," in Society of Photo-Optical Instrumentation Engineers (SPIE) Conference Series, 2016, vol 9905, p. 990528

[19] Heilmann, R.K. et al., "Critical-angle x-ray transmission grating spectrometer with extended bandpass and resolving power $>10,000$," in Society of Photo-Optical Instrumentation Engineers (SPIE) Conference Series, 2016, p. 99051X.

[20] Heilmann, R.K. et al., "Development of a critical-angle transmission grating spectrometer for the International X-Ray Observatory," in Society of Photo-Optical Instrumentation Engineers (SPIE) Conference Series", 2009, p. 74370G.

[21] Heilmann, R.K. et al., "Critical-angle transmission grating spectrometer for high-resolution soft x-ray spectroscopy on the International X-ray Observatory," in Society of Photo-Optical Instrumentation Engineers (SPIE) Conference Series, 2010, p.77321J.

[22] Heilmann, R.K., Bruccoleri, A.R., and Schattenburg, M.L., "High-efficiency blazed transmission gratings for high-resolution soft x-ray spectroscopy," in Society of Photo-Optical Instrumentation Engineers (SPIE) Conference Series, 2015, p. 960314.

[23] Canizares, C. R. et al., "Chandra High Energy Transmission Grating: Design, Fabrication, Ground Calibration, and Five Years in Flight," Publications of the Astronomical Society of the Pacific, vol. 117, pp 1144-1171, 2005

[24] Günther, H.M. et al., "Ray-tracing critical-angle transmission gratings for the X-ray Surveyor and Explorer-size missions," in Society of Photo-Optical Instrumentation Engineers (SPIE) Conference Series, 2016, p. 990556

[25] Bautz, M. et al., "Directly-deposited blocking filters for high-performance silicon X-ray detectors," in 
Society of Photo-Optical Instrumentation Engineers (SPIE) Conference Series, 2016, p. 99054C

[26] Bruccoleri, A. R., Heilmann, R. K., and Schattenburg, M. L., "Fabrication process for 200 nm-pitch polished freestanding ultra-high aspect ratio gratings," J. Vac. Sci. Technol. B 34, 06KD02 (2016).

[27] Gallo, L. et al., "The Canadian Astro-H metrology system," in Space Telescopes and Instrumentation 2012: Ultraviolet to Gamma Ray, J. A. den Herder, T. Takahashi, M. Bautz, Ed., Proc. SPIE 8443, (2012)

[28] Gallo, L. et al., "The Canadian Astro-H metrology system," in Space Telescopes and Instrumentation 2014: Ultraviolet to Gamma Ray, J. A. den Herder, T. Takahashi, M. Bautz, Ed., Proc. SPIE 9144, (2014)

[29] Heilmann, R. K. et al., 2017, "Critical-angle transmission grating technology development for high resolving power soft x-ray spectrometers on Arcus and Lynx" in Society of Photo-Optical Instrumentation Engineers (SPIE) Conference Series, 2017, vol. 10399

[30] Song, J. et al. 2017, "Scanning laser reflection tool for alignment and period measurement of criticalangle transmission gratings," in Society of Photo-Optical Instrumentation Engineers (SPIE) Conference Series, 2017, vol. 10399

[31] Bavdaz, M. et al. 2017, "The Athena telescope and optics status" in Society of Photo-Optical Instrumentation Engineers (SPIE) Conference Series, 2017, vol. 10399

[32] Bianucchi, G. et al. 2017, "Optical integration of SPO mirror modules in the ATHENA telescope" in Society of Photo-Optical Instrumentation Engineers (SPIE) Conference Series, 2017, vol. 10399

[33] Collon, M. et al. 2017, "Development of Athena mirror modules"in Society of Photo-Optical Instrumentation Engineers (SPIE) Conference Series, 2017, 10399-11 